Note: This copy is for your personal non-commercial use only. To order presentation-ready copies for distribution to your colleagues or clients, contact us at www.rsna.org/rsnarights.

Patrick A. Helm, PhD

Peter Caravan, PhD

Brent A. French, PhD

Vincent Jacques, PhD

Luhua Shen, MD

Yaqin Xu, MD

Ronald J. Beyers, PhD

R. Jack Roy, MS

Christopher M. Kramer, MD

Frederick H. Epstein, PhD

\title{
Postinfarction Myocardial
} Scarring in Mice: Molecular MR

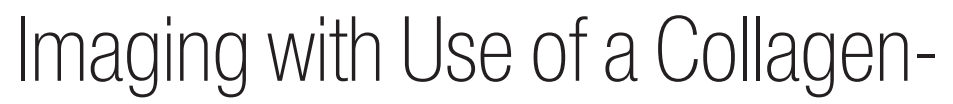
targeting Contrast Agent ${ }^{1}$

Purpose:

Materials and Methods:

Results:

Conclusion:
${ }^{1}$ From the Department of Radiology, University of Virginia, 409 Lane Rd, MR4 Bldg, Room 1186, Charlottesville, VA 22908 (P.A.H., B.A.F., Y.X., R.J.B., R.J.R. C.M.K., F.H.E.); and Epix Pharmaceuticals, Lexington, Mass (P.C., V.J., L.S.). Received June 5, 2007; revision requested August 6; revision received 0ctober 5; final version accepted October 29. Supported in part by National Institutes of Health grant R01 EB 001763. F.H.E. supported by an American Heart Association Established Investigator award. P.A.H. supported by National Institutes of Health grant HL207284. Address correspondence to F.H.E. (e-mail: fredepstein@virginia.edu).
To prospectively evaluate a gadolinium-based collagen-targeting contrast agent, EP-3533, for in vivo magnetic resonance (MR) imaging of myocardial fibrosis in a mouse model of healed myocardial infarction (MI).

All procedures were performed in accordance with protocols approved by the animal care and use committee. MI was induced in eight mice by means of occlusion of the left anterior descending coronary artery followed by reperfusion. Four MR examinations were performed in each animal: one examination before, one examination 1 day after, and two examinations 6 weeks after the MI. For the latter two examinations, electrocardiographically gated inversion-recovery gradient-echo MR images were acquired before and serially (every 5 minutes) after the intravenous injection of either gadopentetate dimeglumine or EP-3533. The image enhancement kinetic properties of the postinfarction scar, normal myocardium, and blood were compared.

Dynamic T1-weighted MR imaging revealed the washout time constants for EP-3533 to be significantly longer than those for gadopentetate dimeglumine in regions of postinfarction scarring (mean, 194.8 minutes \pm 116.8 [standard deviation] vs 25.5 minutes $\pm 4.2 ; P<.05)$ and in normal myocardium (mean, 45.4 minutes \pm 16.7 vs 25.1 minutes $\pm 9.7 ; P<.05)$. Findings on postmortem histologic sections stained for collagen correlated well with EP-3533enhanced areas seen on inversion-recovery MR images. Fifty minutes after EP-3533 injection, the postinfarction scar tissue samples, as compared with the normal myocardium, had a twofold higher concentration of gadolinium.

Use of the gadolinium-based collagen-targeting contrast agent, EP-3533, enabled in vivo molecular MR imaging of fibrosis in a mouse model of healed postinfarction myocardial scarring.

(๑) RSNA, 2008 
ollagen is a highly abundant protein that has an important role in the structural scaffold of connective and most other tissues. In the myocardium, collagen, approximately $85 \%$ of which is type $\mathrm{I}$, in its native concentration forms a dynamic construction that not only supports and aligns myocytes into an energetically favorable architecture but also transmits active and passive forces throughout its structure (1-4).

In wound healing, collagen synthesis is upregulated. Fibrosis is the development of excess fibrous connective tissue-largely type I collagen-as a result of a reparative or reactive process and is a characteristic of many cardiovascular and other abnormalities (5-8). After myocardial infarction (MI), wound healing results in the generation of fibrotic scars and contributes to postinfarction left ventricular (LV) remodeling. Molecular imaging of collagen could yield valuable insight into the progression or regression of fibrosis.

MR imaging could be an ideal modality for detecting fibrosis owing to its high soft-tissue contrast and high spatial resolution. Collagen is present at relatively high concentrations, which may be detectable with use of a collagenspecific MR contrast agent. We hypothesized that a collagen-targeting gadolin-

\section{Advances in Knowledge}

- The gadolinium-based collagentargeting contrast agent, EP-3533, enabled in vivo molecular MR imaging of fibrosis in a mouse model of healed postinfarction myocar dial scarring.

- Dynamic T1-weighted MR imaging revealed significantly longer washout time constants for EP3533 compared with the washout time constants for gadopentetate dimeglumine in regions of postinfarction scarring $(>50$ minutes vs 25.5 minutes \pm $4.2, P<.05)$ and in normal myocardium (45.4 minutes \pm 16.7 vs 25.1 minutes $\pm 9.7, P<$ $.05)$. ium-based MR contrast agent would generate persistent high enhancement of fibrotic lesions. Moreover, postinfarction myocardial scar tissue represents a clinically relevant animal model for evaluating a collagen-targeting contrast agent. Thus, the purpose of our study was to evaluate a gadoliniumbased collagen-targeting contrast agent (EP-3533; Epix Pharmaceuticals, Lexington, Mass) for in vivo MR imaging of myocardial fibrosis in a mouse model of healed MI.

\section{Materials and Methods}

All in vivo surgical and imaging procedures were performed in accordance with protocols approved by the University of Virginia animal care and use committee. Epix Pharmaceuticals supplied the EP-3533 and provided financial support for this research. The authors who are not employees of Epix Pharmaceuticals had control of the inclusion of any data and information that might represent a conflict of interest for those authors who are employees of that company.

\section{Mouse Infarct Model}

One author (Y.X.) with 3 years experience in animal surgery induced $\mathrm{MI}$ in eight male C57BL/6 mice (Jackson Laboratory, Bar Harbor, Me) with a mean age of 12 weeks \pm 3 (standard deviation) by occluding the left anterior descending coronary artery and then reestablished perfusion. Mice were anesthetized by means of an intraperitoneal injection of $100 \mu \mathrm{g}$ of pentobarbital sodium per gram of body weight, and thoracotomy was performed. The pericardium was removed, and the left anterior coronary artery was occluded with a 7.0 silk suture for 60 minutes; perfusion

\section{Implication for Patient Care}

- Although still in the preclinical stage, EP-3533 may be useful for detecting and evaluating a broad array of human diseases, including myocardial, hepatic, and pulmonary fibroses. was then reestablished. In this model, which is well established, scar formation is complete 28 days after the MI $(9,10)$.

\section{Collagen-targeting Contrast Agent}

The collagen-targeting contrast agent, EP-3533, was designed by appending gadopentetate dimeglumine moieties for positive contrast material-enhanced imaging to a collagen-specific peptide for molecular targeting. The peptide consists of a cyclic 10-amino acid peptide that is cyclized via a disulfide bond between two cysteines with three flanking residues at both the $\mathrm{C}$ terminus and the $\mathrm{N}$ terminus. The peptide contains three primary amines ( $\mathrm{N}$ terminus and two lysine side chains), and these were used to append three gadopentetate dimeglumine moieties via a thiourea linkage. The structure, full synthesis, and synthetic characterization of EP-3533 have been described previously (11). In vitro experiments have revealed that EP-3533 binds reversibly to mouse type I collagen $\left(K_{d}=1.8 \mu \mathrm{M}\right)$ with high stoichiometric binding properties $(\mathrm{N}>7$ equivalent binding sites) (11).

The relaxivity of EP-3533 was assessed (P.A.H., P.C., V.J.) at 0.47, 1.41 , and $4.70 \mathrm{~T}$ by measuring the $\mathrm{T} 1$

\section{Published online before print 10.1148/radiol.2473070975 \\ Radiology 2008; 247:788-796 \\ Abbreviations: \\ $\mathrm{CNR}=$ contrast-to-noise ratio \\ $\mathrm{IR}=$ inversion recovery \\ $\mathrm{LV}=$ left ventricle \\ $\mathrm{MI}=$ myocardial infarction}

\section{Author contributions:}

Guarantors of integrity of entire study, P.A.H., F.H.E.; study concepts/study design or data acquisition or data analysis/interpretation, all authors; manuscript drafting or manuscript revision for important intellectual content, al authors; manuscript final version approval, all authors; literature research, P.A.H., P.C., F.H.E.; experimental studies, P.A.H., P.C., B.A.F., V.J., L.S., Y.X., R.J.R., C.M.K., F.H.E.; statistical analysis, P.A.H., P.C., R.J.B., F.H.E.; and manuscript editing, P.A.H., P.C., B.A.F., V.J., F.H.E.

See Materials and Methods for pertinent disclosures. 
relaxation times of phosphate-buffered saline samples ( $\mathrm{pH} 7.4$ ) containing increasing amounts of EP-3533. Since EP3533 comprises three $\mathrm{Gd}^{3+}$ ions, relaxivity was expressed per gadolinium ion and per EP-3533 molecule (Table 1). The 0.47-T (Bruker NMS120; Bruker Optics, The Woodlands, Tex) and 1.41-T (Bruker mq60; Bruker Optics) spectrometers used were equipped with water-heated coils, which enabled us to maintain the temperature of the samples at $37^{\circ} \mathrm{C}$. The larger $(4.70-\mathrm{T})$ spectrometer was not equipped with heated coils, so measurements were made at $25^{\circ} \mathrm{C}$. Relaxivity ( 1 1) was calculated as the slope of a plot of R1 (1/T1) versus concentration. In Table 1 , these measurements are compared with previously published gadopentetate dimeglumine relaxivity data acquired at matching field strengths and at temperatures of $37^{\circ}-40^{\circ} \mathrm{C}(12)$.

\section{MR Imaging}

Imaging was performed (P.A.H., R.J.R.) by using a 4.70-T MR imaging system (Varian, Palo Alto, Calif). Four MR examinations were performed in each mouse $(n=8)$. The first examination was performed before the induced MI for baseline assessment of cardiac function; the three remaining examinations were performed after the MI. For each MR examination, the mouse was anesthetized with isoflurane ( 1 vol\% in oxygen). Also, for each examination, three pediatric electrocardiographic leads were attached to shaved limbs and a rectal temperature probe was placed. The electrocardiogram and core body temperature were recorded during imaging by using a SAII Model 1025 monitoring and gating system (Small Animal Instruments, Stony Brook, NY). A mean temperature of $37.0^{\circ} \mathrm{C} \pm 0.5$ (standard deviation) was maintained by using hot water circulating through tubes placed in contact with the animals during imaging. For post-MI day 1 assessment of infarct size, an indwelling intraperitoneal catheter was placed for injection of gadopentetate dimeglumine (Magnevist; Berlex Laboratories, Wayne, NJ).

For all MR examinations, cardiac function was assessed by using a

\section{Table 1}

\section{Relaxivity of EP-3533 and Gadopentetate Dimeglumine}

\begin{tabular}{|c|c|c|c|}
\hline \multirow[b]{2}{*}{ Field Strength (T) } & \multicolumn{2}{|c|}{ EP-3533 $\left(\mathrm{L} \cdot \mathrm{mmol}^{-1} \cdot \mathrm{sec}^{-1}\right)$} & \multirow{2}{*}{$\begin{array}{l}\text { Gadopentetate Dimeglumine } \\
\left(\mathrm{L} \cdot \mathrm{mmol}^{-1} \cdot \mathrm{sec}^{-1}\right) \text {, per } \\
\text { Gadolinium lon* }\end{array}$} \\
\hline & Per Gadolinium Ion & Per EP-3533 Molecule & \\
\hline 0.47 & $18.7^{\dagger}$ & $56.2^{\dagger}$ & 3.4 \\
\hline 1.41 & $16.1^{\dagger}$ & $48.4^{\dagger}$ & 3.3 \\
\hline 4.70 & $5.4^{\dagger}$ & $16.2^{\dagger}$ & 3.2 \\
\hline
\end{tabular}

Note.-Data are relaxivity values.

* Reference 12.

$\dagger P<.05$ for comparison with gadopentetate dimeglumine values.

\section{Table 2}

\begin{tabular}{|c|c|c|c|}
\hline Assessed Parameter* & Baseline & 1 Day after Ml & 6 Weeks after $\mathrm{Ml}$ \\
\hline Heart rate (beats per minute) & $451 \pm 41$ & $584 \pm 65^{\dagger}$ & $482 \pm 13$ \\
\hline LV EDV $(\mu \mathrm{L})$ & $42 \pm 5$ & $52 \pm 7$ & $74 \pm 16^{\dagger}$ \\
\hline $\operatorname{LV}$ ESV $(\mu \mathrm{L})$ & $17 \pm 2$ & $31 \pm 6^{\dagger}$ & $49 \pm 14^{\dagger}$ \\
\hline Ejection fraction (\%) & $59 \pm 2$ & $39 \pm 6^{\dagger}$ & $34 \pm 6^{\dagger}$ \\
\hline Infarct fraction (\%) & 0 & $40 \pm 5^{\dagger}$ & $32 \pm 5^{\dagger \neq}$ \\
\hline
\end{tabular}

Note.-All except the baseline infarct fraction are mean values \pm standard deviations.

${ }^{*} \mathrm{EDV}=$ end-diastolic volume, $\mathrm{ESV}=$ end-systolic volume.

${ }^{\dagger} P<.05$ for comparison with baseline value.

$\ddagger P<.05$ for comparison between value obtained 1 day and value obtained 6 weeks after MI.

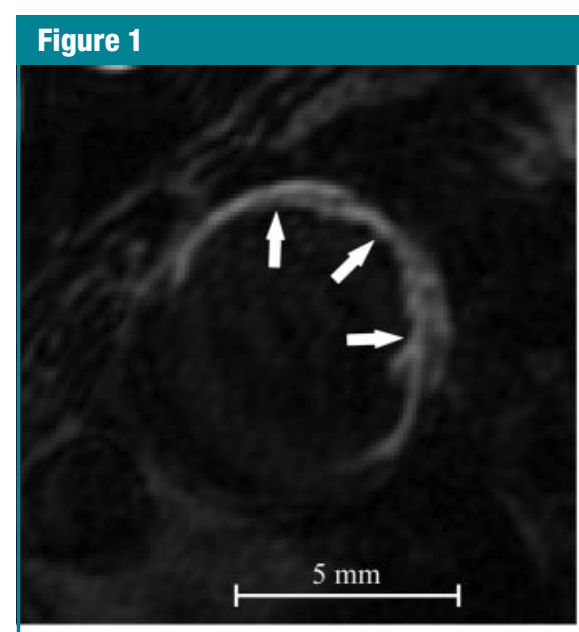

a.

Figure 1: Midventricular short-axis IR gradient-echo MR images (7.1/3.0/430) of LV obtained in the same heart and at a similar location (a) 40 minutes after EP-3533 injection and (b) 15 minutes after gadopentetate dimeglumine injection. Binding of EP-3533 to collagenous scar tissue (arrows) after washout from the blood pool and from the normal myocardium enables accurate differentiation between scar tissue and blood and between scar tissue and normal myocardium. IR imaging with gadopentetate dimeglumine-induced delayed enhancement confirms the region of postinfarction scarring. 
short-axis stack (six to eight sections) of black blood cine images acquired by using a double inversion-recovery (IR) gradient-echo pulse sequence $(10,13)$. The following parameters were used: repetition time msec/echo time msec/ inversion time msec, 8/3.7/R-R interval (approximately $120 \mathrm{msec}$ ); matrix, $128 \times 128$; field of view, $2.56 \times 2.56$ $\mathrm{cm}$; section thickness, $1 \mathrm{~mm}$; flip angle, $20^{\circ}$; and four signals acquired. Double IR pulses were applied at the detection of every second $\mathrm{R}$ wave, and image data were acquired during successive heart beats by using a multiphase acquisition in which 12-14 lines with the same phase-encoding value were acquired. The total acquisition time for a single section was approximately 4 minutes.
On post-MI day 1, gadopentetate dimeglumine was injected intraperitoneally and short-axis electrocardiographically gated multisection IR gradient-echo MR images were acquired from the base to the apex of the heart in two separate acquisitions $(14,15)$. A nonselective inversion radiofrequency pulse was applied after the detection of an $\mathrm{R}$ wave, and after a delayed inversion time and the detection of an electrocardiographic trigger, one line of raw data for three different sections was acquired. We set the delay between detection of the electrocardiographic trigger and application of the inversion pulse to achieve the specified inversion time (430 msec) for the second of the three sections. Since three sections were acquired per examination, the first and third sections had a slightly different inversion time (430 msec minus repetition time and 430 msec plus repetition time, respectively) and hence a slightly different cardiac phase. This relatively small degree of variability was accepted for the purpose of decreasing the imaging time by a factor of three compared with the imaging time for single-section imaging. The following parameters were used: 7.1/3.0 (repetition time msec/echo time msec); field of view, $2.56 \times 2.56 \mathrm{~cm}$; matrix, $128 \times$ 128; flip angle, $90^{\circ}$; section thickness, 1 $\mathrm{mm}$; intersection gap, $1 \mathrm{~mm}$; two signals acquired; and time between inversions, approximately $2000 \mathrm{msec}$.

Two scar imaging examinations were conducted 6 weeks after MI, allowing adequate time for scar formation

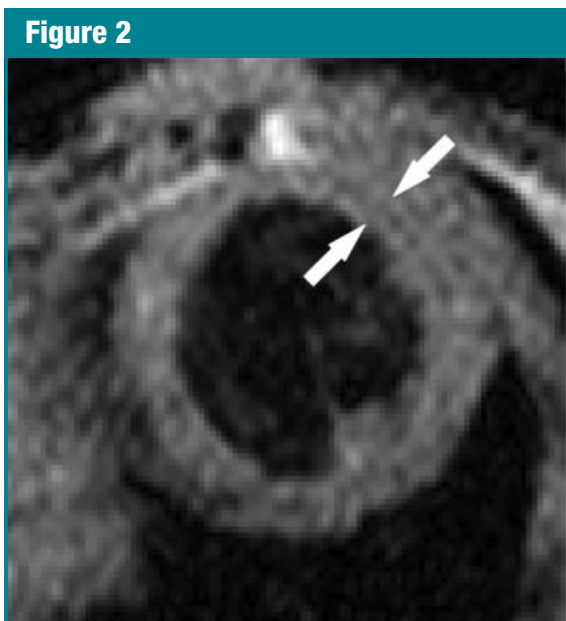

a.

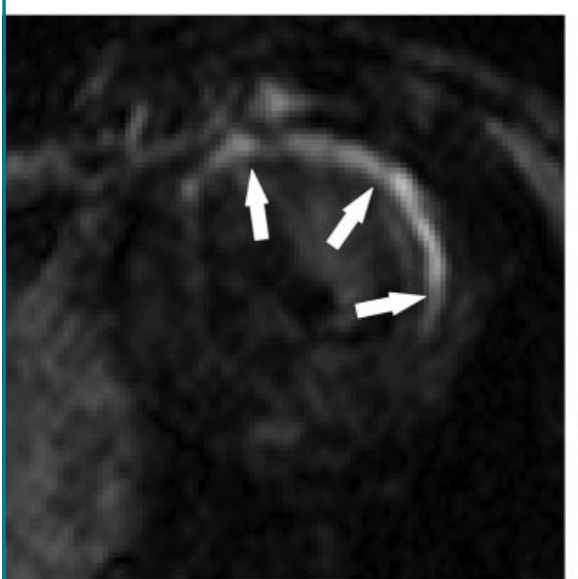

d.

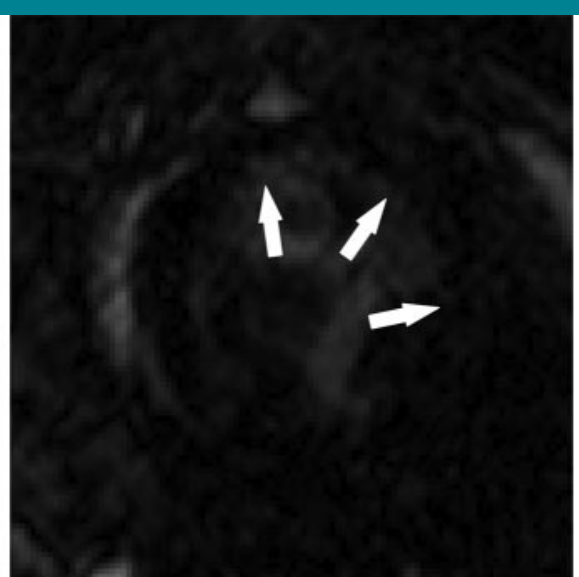

b.

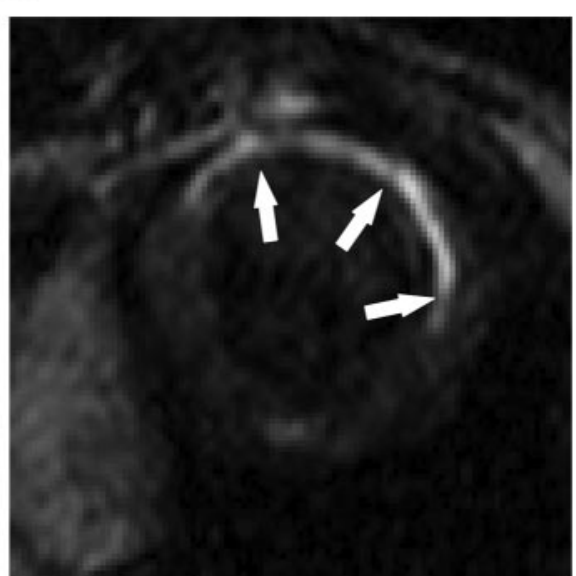

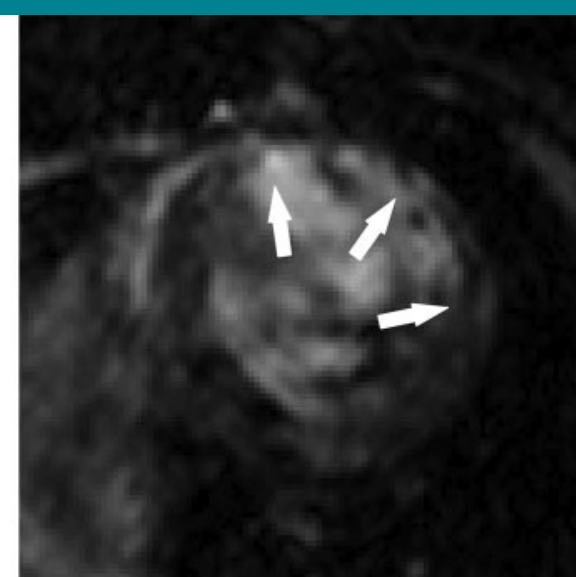

C.

Figure 2: (a) Midventricular short-axis blackblood gradient-echo MR image (8/3.7, 20 flip angle) of the LV obtained 6 weeks after experimental MI shows thinning of the scarred anterior wall (arrows). (b-e) IR MR images (7.1/3.0/430) of the same section obtained (b) before and (c) 5 minutes, (d) 20 minutes, and (e) 35 minutes after EP-3533 injection show EP3533 kinetic properties, including persistent enhancement of the scar (arrows). (c) Shortly after the injection, the blood pool, normal myocardium, and scarred tissue are enhancing. As time progresses, enhancement of the blood pool and the normal myocardium (septum and inferior wall) starts to diminish until the last frame (e), in which only the scar (LV anterior and lateral walls) remains strongly enhanced. 
(9). The MR imaging parameters used were almost identical to those used at imaging on post-MI day 1; however, instead of administering the contrast agent by means of intraperitoneal injection, we used indwelling tail vein catheters to eliminate potential dampening of the enhancement kinetic properties. Specifically, we used a 30-gauge needle attached to polyethylene-10 tubing (inner diameter, $100 \mu \mathrm{m}$ ), which extended outside the bore of the magnet and was initially filled with a heparin-saline solution (10 IU/mL heparin) to prevent clotting. Conventional nontargeting gadopentetate dimeglumine, $0.2 \mathrm{mmol}$ per kilogram of body weight, was injected during the first MR examination, and $0.025 \mathrm{mmol} / \mathrm{kg}$ EP-3533 was injected during the second examination; these two examinations were performed at least 2 days apart to ensure complete washout of gadopentetate dimeglumine. The $0.025 \mathrm{mmol} / \mathrm{kg}$ dose of EP-3533 was chosen on the basis of pilot biodistribution studies in healthy animals (11) and the relaxivity of the agent at $4.70 \mathrm{~T}$. Initial imaging examinations revealed that this dose yielded a concentration of gadolinium sufficient to enhance normal and infarcted myocardia. On the basis of relaxivity differences, the higher gadopentetate dimeglumine dose $(0.2 \mathrm{mmol} / \mathrm{kg})$ was expected to induce enhancement similar to that generated with EP-3533. This dose is also typically used for clinical viability imaging (16). A stack of IR images was acquired before and serially (every 5 minutes) after contrast agent injection for 50 minutes. Eight mice were injected with EP-3533 and gadopentetate dimeglumine 6 weeks after MI; however, only seven hearts were used in the image analysis, because one mouse had poor electrocardiographic gating during MR imaging.

\section{Image Analysis}

LV volumes and ejection fractions were estimated from segmented cine images. Regions of interest with average sizes of $4.6 \mathrm{~cm}^{2}$ for blood pool, $2.8 \mathrm{~cm}^{2}$ for scarred myocardium, and $3.2 \mathrm{~cm}^{2}$ for nonscarred myocardium were delineated from the IR images (P.A.H., 10

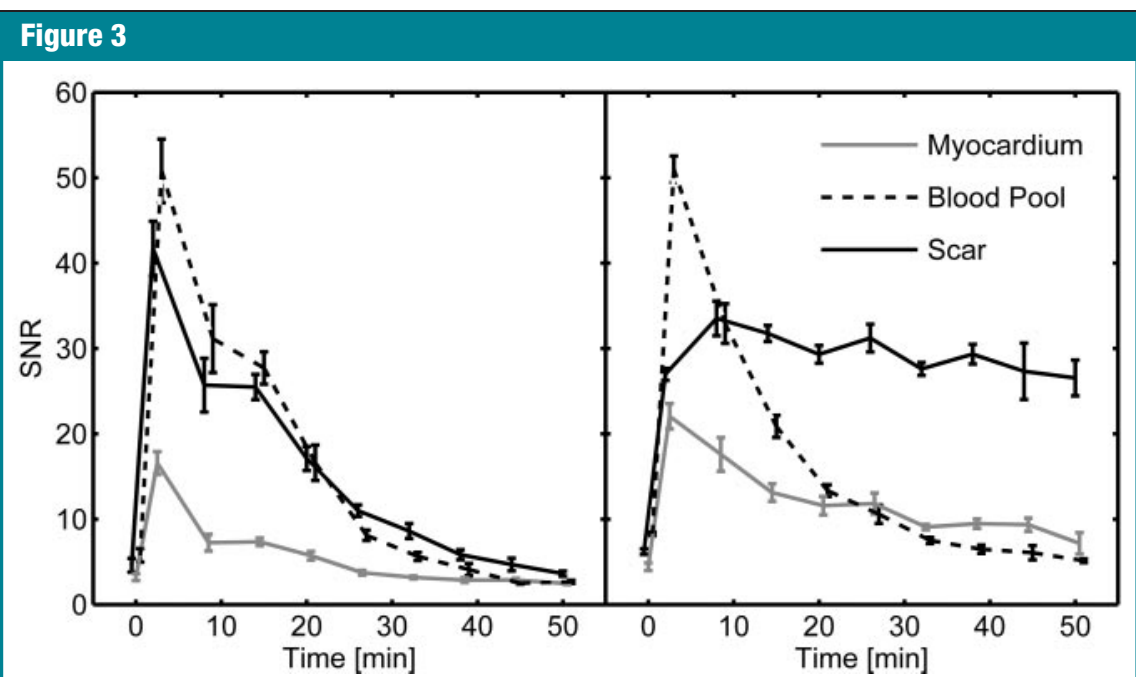

Figure 3: Plots of mean signal-to-noise ratio (SNR) (seven hearts) for blood, normal myocardium, and scar tissue as a function of time after injection of gadopentetate dimeglumine (left) and EP-3533 (right) predominately indicate prolonged EP-3533 enhancement of the scar compared with the duration of gadopentetate dimeglumine enhancement. The plotted data also indicate greater enhancement in and slower washout from the normal myocardium after an injection of EP-3533 compared with these parameters after an injection of gadopentetate dimeglumine.

\section{Table 3}

Washout Time Constants for Gadopentetate Dimeglumine and EP-3533

\begin{tabular}{lll} 
Anatomic Area & $\begin{array}{l}\text { Gadopentetate } \\
\text { Dimeglumine }\end{array}$ & EP-3533 \\
\hline Blood & $17.9 \pm 4.2$ & $15.0 \pm 2.7$ \\
Normal myocardium & $25.1 \pm 9.7$ & $45.4 \pm 16.7^{\dagger}$ \\
Scarred myocardium & $25.5 \pm 4.2$ & $194.8 \pm 116.8^{\star \dagger}$ \\
\hline
\end{tabular}

Note.-Data are mean washout time constants (in minutes) \pm standard deviations

* The time constant was longer than the imaging period.

${ }^{\dagger} P<.05$ for comparison with gadopentetate dimeglumine value.

years experience in cardiac MR imaging). Signal-to-noise ratio and contrastto-noise ratio (CNR) were computed for each region of interest. MR signal enhancement was divided by the background image noise, and the variation in background noise throughout each image was verified to be minimal. Region-of-interest enhancement was not referenced to another tissue because all tissues have some concentration of collagen. We quantified the kinetic properties of contrast agent washout in each region of interest by fitting an exponential function to the decay portion of the signal-to-noise ratio-time curves.
Comparison between Histologic Analysis and MR Imaging Findings

Histologic analysis of scarred tissue is invasive, and the fixation process does not permit quantification of gadolinium concentration in tissue. Therefore, after all MR examinations were completed, three mice were randomly chosen for histologic analysis by two authors (P.A.H., Y.X., 3 and 5 years experience in cardiac pathology, respectively), and the remaining five animals were used for gadolinium quantification. After the three mice were euthanized, their hearts were explanted, thoroughly washed in saline 
solution, and fixed in an isotonic solution of formaldehyde ( $3 \%$ by volume) for 12 hours at $3^{\circ} \mathrm{C}$. The hearts were then washed in phosphate-buffered saline and stored in 70\% alcohol before being embedded in paraffin. Each heart was cut into 10 - $\mu$ m-thick sections from the base to the apex and stained with picrosirius red dye (17), which enhances the contrast between myocytes and collagen. Three shortaxis sections (base, midventricle, and

\section{Figure 4}

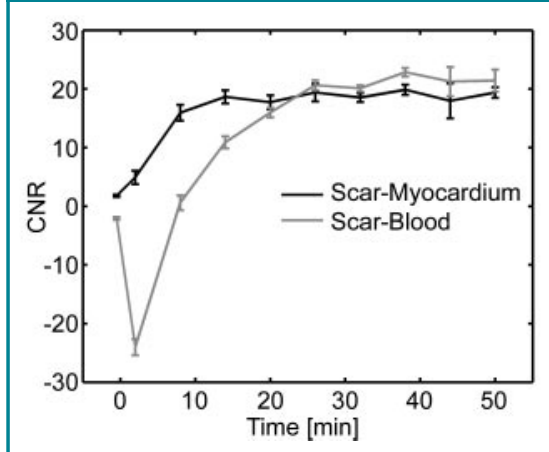

Figure 4: Plots of mean CNR $(n=7)$ between scar tissue and normal myocardium and between scar tissue and blood as a function of time after EP-3533 injection. The maximal CNR between scarred tissue and blood and between scarred tissue and normal myocardium occurred 15 minutes after the injection, and the high CNR persisted for at least 50 minutes.

\section{Figure 5}

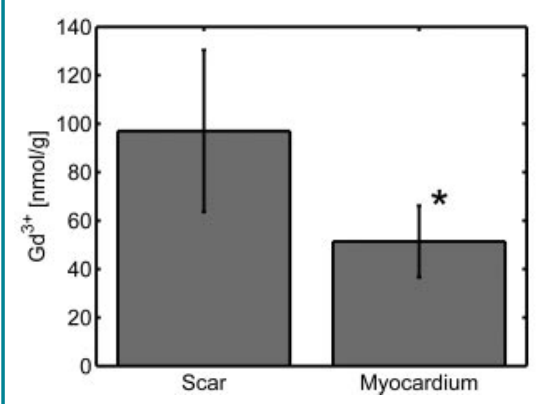

Figure 5: Bar graph shows quantification of gadolinium concentration measured by using inductively coupled plasma mass spectrometry in scarred versus normal myocardium 50 minutes after injection of EP-3533. * $=P<.05$. apex) per heart were identified by using anatomic landmarks that matched corresponding positions on three MR images. (Only those sections containing both scarred and nonscarred tissue were chosen.) The circumferential extent of scarring depicted on the EP3533-enhanced MR images was measured (in degrees) and with use of linear correlation compared (P.A.H.) with the circumferential extent of scarring stained in picrosirius red on the histologic sections.

\section{Gadolinium Quantification}

Gadolinium quantification must be performed while the contrast agent is still present in the target tissue. Therefore, the remaining five mice were euthanized 50 minutes after EP3533 injection, and their hearts were explanted, thoroughly washed in saline solution, and grossly divided into two sections-scarred tissue versus nonscarred tissue- by means of visual detection of white epicardium, which is associated with scarring. The samples were weighed and frozen for quantification of gadolinium concentration. Two authors (P.C., V.J.) subsequently dissolved each sample in nitric acid and measured the gadolinium concentration (in nanomoles per gram) by using inductively coupled plasma mass spectrometry with terbium as an internal standard. In addition, blood and kidney samples were taken from two of the mice 50 minutes after the injection, and the gadolinium concentrations in these tissues were assessed in a similar fashion.

\section{Statistical Analyses}

Our experiments were powered for the detection of slower EP-3533 washout from scarred tissue compared with gadopentetate dimeglumine washout. Initial data from two mice suggested a mean difference in washout time between EP-3533 and gadopentetate dimeglumine of 25 minutes \pm 5 ; thus, an experimental sample size of eight animals was chosen for $99 \%$ confidence. Significant differences in regional signal-to-noise ratios, washout time constants, and gadolinium concentrations were deter- mined by using a two-tailed Student $t$ test, and for serial measurements of LV function, one-way repeated measures analysis of variance was used. The Friedman repeated measures analysis of variance on ranks was used to analyze data that did not satisfy the normality criteria. For assessment of the linear correlation between circumferential extent of scarring measured with EP-3533-enhanced MR imaging and that measured at histologic analysis, Pearson correlation coefficients were calculated. Two authors (P.A.H., F.H.E.) performed all statistical analyses by using commercially available software (SigmaStat 3.1; Systat Software, Point Richmond, Calif). $P<.05$ was considered to indicate significant differences. All data are reported as means \pm standard deviations.

\section{Results}

\section{Relaxivity, Ejection Fraction, and Infarct Size}

EP-3533 had significantly higher relaxivity than did gadopentetate dimeglumine at each field strength $(P<.05)$ and thus greater sensitivity for the detection of collagen (Table 1). All hearts showed a significant reduction in ejection fraction as a result of the MI (Table 2) compared with baseline values $(P<.001)$. The mean $\mathrm{LV}$ volume fraction that was infarcted, as indicated by post-MI day 1 delayed gadolinium-enhanced imaging findings, was $40 \% \pm 5.3$, indicating that all of the coronary occlusions resulted in large MIs.

\section{Kinetic Properties of EP-3533 and Gadopentetate Dimeglumine}

Images enhanced with gadopentetate dimeglumine and EP-3533 are shown in Figures 1 and 2. In the mice injected with gadopentetate dimeglumine, the signal-to-noise ratio of the blood pool and the scarred tissue was increased 5 minutes after injection and washout from both regions followed 10-30 minutes later. Mild early enhancement of the normal myocardium was also seen. EP-3533 showed considerably different kinetic properties, particu- 


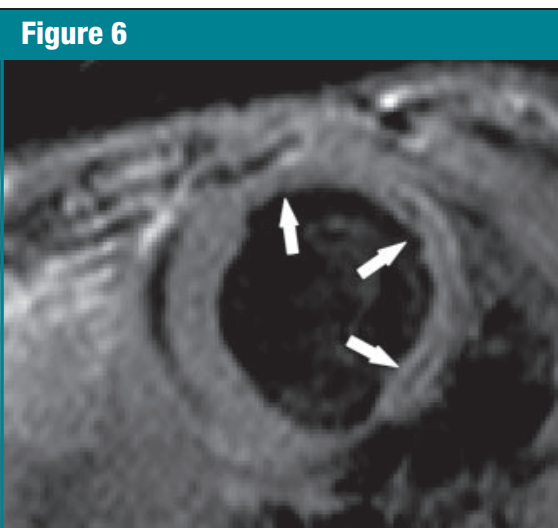

a.

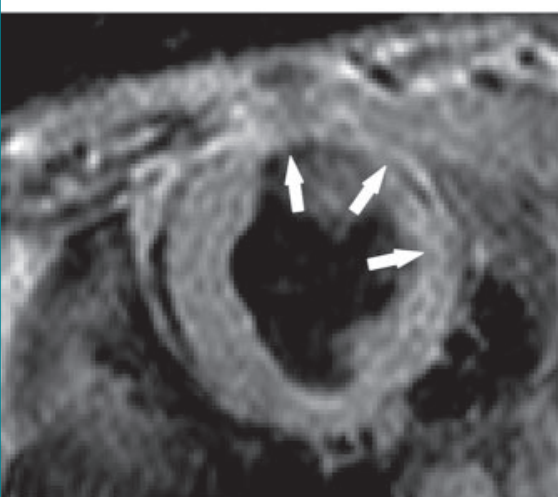

d. area of scarring. (a, d) Standard anatomic MR images acquired by using a double IR gradient-echo sequence (8.0/3.7/R-R interval). (b, e) Regions of contrast enhancement on midventricular short-axis MR images (7.1/3.0/430) of the LV at two section locations obtained 40 minutes after EP-3533 injection correlate closely with (c, f) photomicrographs of picrosirius red-stained tissue sections shown at nine times their original size.

larly in scarred tissue and normal myocardium (Fig 3, Table 3). Like gadopentetate dimeglumine, EP-3533 caused immediate enhancement of the blood pool, with washout occurring within a mean decay time constant of 15 minutes \pm 2.7 (Fig 4). Strong enhancement of the scarred tissue peaked 10 minutes after injection, and enhancement of the scar persisted for at least 50 minutes. Normal myocardium showed an intermediate level of early enhancement with EP-3533, and the washout of EP-3533 from the myocardium was significantly longer than that of gadopentetate dimeglumine (mean first-order time constants, 45.4 minutes \pm 16.7 and 25.1 minutes \pm 9.7, respectively; $P<.05$ ).

The scar-blood CNR was initially negative owing to the strong enhance-

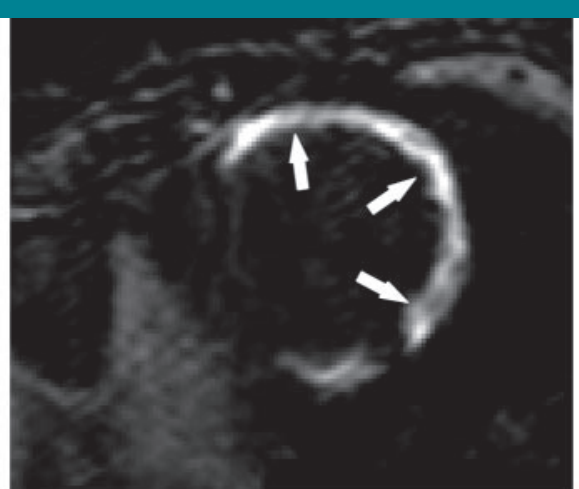

b.
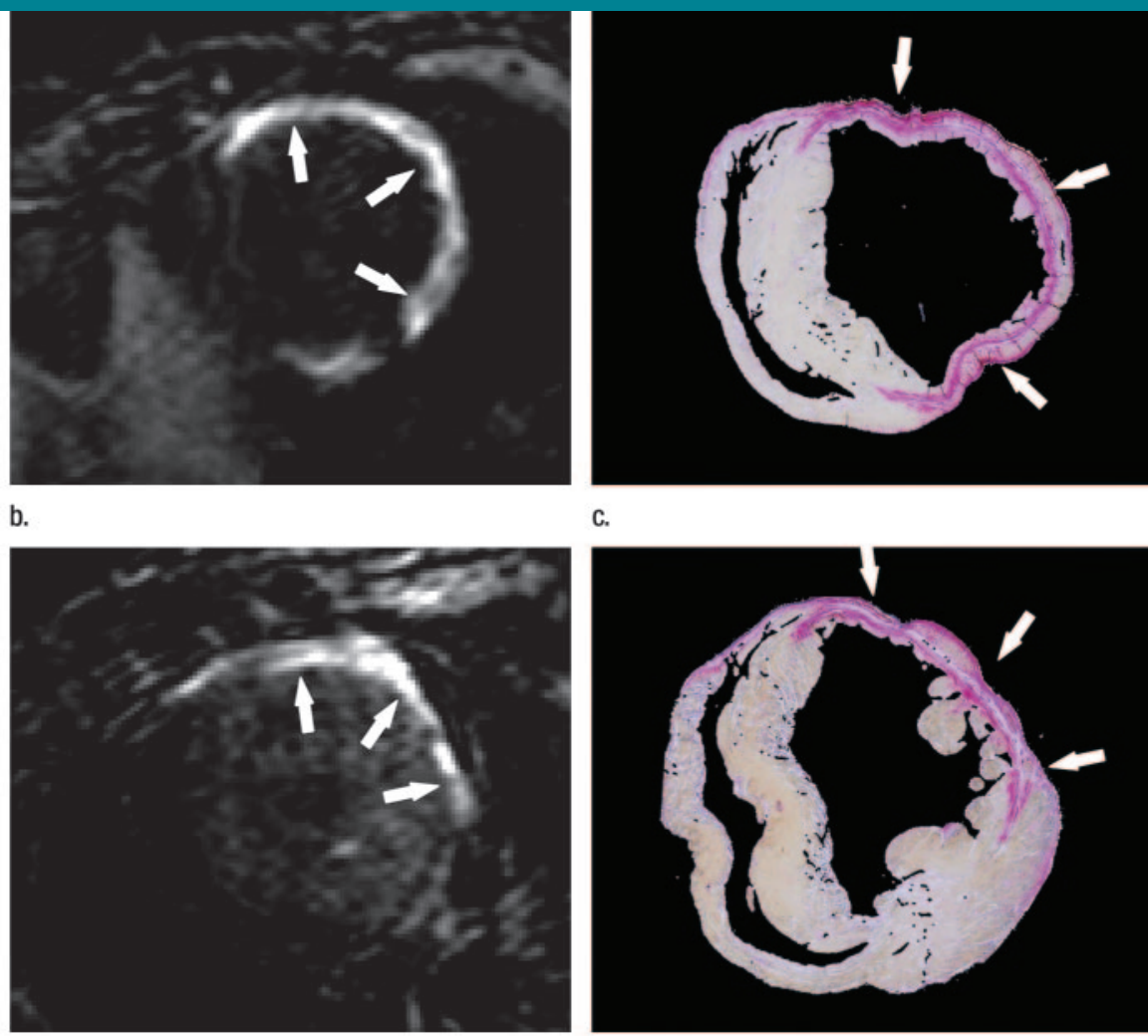

c.

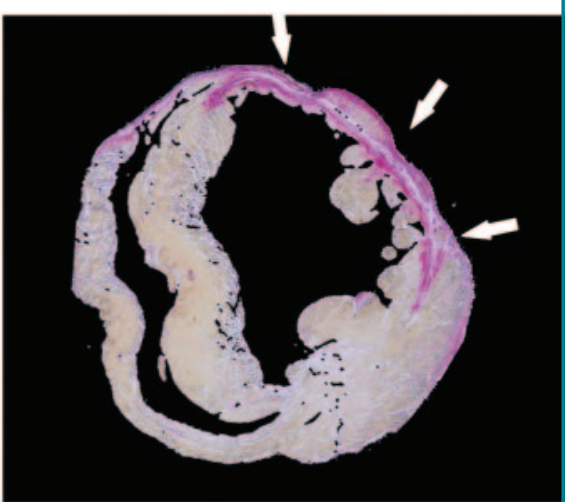

f. ment of the blood after EP-3533 injection; it then increased as the scarred tissue enhanced and EP-3533 washed out of the blood. Thirty-five minutes after injection, the CNR peaked at approximately 20 . The scar-normal myocardium CNR also increased with time, peaking at approximately 18 at 15 minutes after injection.

\section{Gadolinium Quantification}

Five of the mice injected with EP-3533 were euthanized 50 minutes after the injection to determine regional $\mathrm{Gd}^{3+}$ concentrations (Fig 5). On average, the scarred tissue showed a twofold increase in gadolinium concentration compared with the normal myocardium (97.8 nmol/g $\pm 33.4 \mathrm{vs} 50.4 \mathrm{nmol} / \mathrm{g} \pm$ 14.8). Blood and kidney samples (two each) taken 50 minutes after injection showed mean gadolinium concentrations of $21.2 \mathrm{nmol} / \mathrm{g} \pm 9.4$ and 338.8 $\mathrm{nmol} / \mathrm{g} \pm 30$, respectively.

\section{Comparison of Imaging and Histologic Findings}

For all three hearts sectioned and stained for collagen, there was a quantitative correlation $\left(R^{2}=0.97\right)$ between the circumferential extent of EP-3533induced strong enhancement on the IR images at 40 minutes and the extent of picrosirius red staining of scarred tissue at apical, midventricular, and basal locations (Figs 6, 7). Planimetry with use of the EP-3533-enhanced images acquired 6 weeks after the MI revealed the scar size to be a mean $32 \% \pm 5$ of the LV mass, which is significantly smaller $(P<.05)$ than the infarct size measured on post-MI day 1 (Table 2). 


\section{Discussion}

An important challenge in molecular MR imaging is that of achieving the concentration of contrast agent required (in micromoles per liter) to affect signal intensity (18). Collagen is a high-concentration target-about $5 \%$ of the dry weight of normal human myocardium is collagen (19) - and in fibrotic tissue such as scar, this concentration is increased (20). Thus, the concentration of collagen is $10 \mathrm{~s}$ of nanomoles per gram (nanomoles per gram $\approx$ micromoles per liter since tissue density $\approx 1$ $\mathrm{g} / \mathrm{mL}$ ) in the normal myocardium and is considerably higher in scarred tissue. Because collagen is an extracellular protein, the concentration of collagen in the extracellular space is high. Also, collagen is a good target for fibrosis in general and for MI in particular because, unlike the expression of some cell surface receptors, the expression of collagen is fairly static and prevalent. Furthermore, the extracellular location of collagen makes it accessible because the contrast agent does not need to cross cell membranes. Our study results demonstrate, in a model of chronic MI, that the collagen-targeting contrast agent, EP-3533, becomes sufficiently concentrated in the extracellular space and is effective for in vivo imaging of fibrosis.

\section{Figure 7}

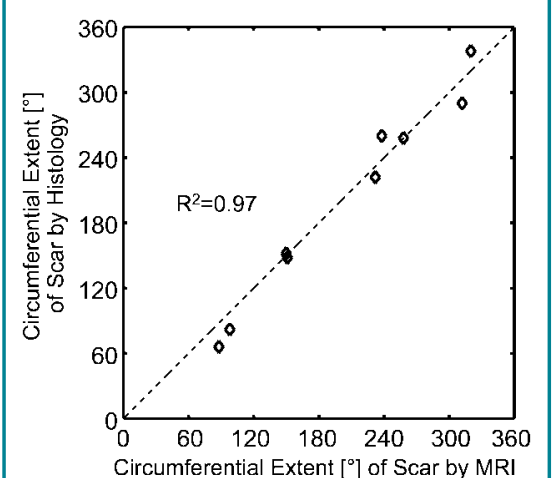

Figure 7: Graph shows correlation between circumferential extent of scarring (in degrees) at EP-3533-enhanced MR imaging and circumferential extent of scarring on picrosirius red-stained histologic sections. Individual measurements $(\diamond)$ were performed at apical, midventricular, and basal locations in three hearts
In our study, EP-3533 enhancement of myocardial scar tissue was assessed with in vivo MR imaging and compared with the enhancement induced by gadopentetate dimeglumine (positive control contrast agent) in the same animal. In vivo MR images were also compared with ex vivo picrosirius red dye-stained tissue, and the gadolinium concentration in scarred tissue, normal myocardium, and blood was compared. Gadopentetate dimeglumine was used as a positive control contrast agent for two reasons: First, delayed imaging after injection of the interstitial contrast agent, gadopentetate dimeglumine, has been shown to be effective for identifying post-infarct scarring in both clinical studies and mouse models $(15,21)$. With imaging of the same animal 2 days apart and well after the infarction, the gadopentetate dimeglumine-enhanced MR image enables clear delineation of the infarction zone to use for comparison with the EP-3533- enhanced images. Second, the mechanism of delayed enhancement of the infarct with gadopentetate dimeglumine is related to a difference in washout rates between scarred tissue and normal myocardium and to the larger volume fraction of $\mathrm{ex}^{-}$ tracellular space in scarred tissue than in normal tissue. It was important for us to distinguish, if possible, between the enhancement generated by the molecular targeting of EP-3533 and the conventional delayed enhancement effect.

The kinetic properties of EP-3533 enhancement are notably different from those of gadopentetate dimeglumine enhancement. At comparison of signal-tonoise ratio versus time, the washout of EP-3533 from the scar and from the normal myocardium was significantly slower than the washout of gadopentetate dimeglumine, while the rates of EP-3533 and gadopentetate dimeglumine clearance from the blood pool were similar. These properties result in a scar-blood CNR and scar-normal myocardium CNR that initially increase with time and then remain high for at least 50 minutes. We attribute the retention of EP-3533 in both the normal myocardium and the scarred tissue to the collagen-binding properties of EP-
3533. The greater enhancement and retained contrast in the scarred tissue than in the normal myocardium may have been due to the higher collagen content and/or the greater extracellular volume in the scarred tissue.

Delineation of scarred tissue with EP-3533-enhanced MR imaging correlated well with picrosirius red staining for collagen. The scarred tissue identified on the gadopentetate dimeglumineenhanced MR images was always seen on the corresponding EP-3533-enhanced images. The conspicuity of scarred tissue on the EP-3533-enhanced images was greater at later time points because of the washout of this agent from both the blood and the viable myocardium. The conspicuity of scarred tissue was greater on the EP-3533-enhanced images than on the gadopentetate dimeglumine-enhanced images at later time points; however, it may be possible to improve the CNR between blood and scarred tissue at gadopentetate dimeglumine-enhanced imaging by exploiting the properties of flowing blood. Finally, the decrease in size of an older infarct compared with the size of an acute MI is consistent with contrastenhanced imaging findings in patients before and those in patients after infarct healing (22).

Variations in enhancement dynamics may be attributed to key design attributes of EP-3533. Collagen targeting is fast because the molecular size of EP3533 (about $5 \mathrm{kDa}$ ) allows it to rapidly access and diffuse into the interstitial space. To achieve rapid targeting, we used a short 16 -amino acid peptide that has a high affinity for mouse type I collagen, which has only a $4 \%$ factor of divergence from human type I collagen without the large size of an antibody. There are multiple sites per collagen molecule to which EP-3533 can bind (ie, the bound probe-to-target ratio is high). In addition, EP-3533 contains three gadopentetate dimeglumine moieties that have higher relaxivities than discrete gadopentetate dimeglumine. This combination of affinity, high target concentration, and increased gadolinium payload and relaxivity enables persistent positive scar tissue contrast at T1- 
weighted MR imaging. The molecular size of the contrast agent also results in relatively fast blood clearance, which results in further contrast between the scarred tissue and the blood pool. Together, these factors contribute to a twofold increase in gadolinium concentration in the scarred tissue relative to the normal myocardium and a fourfold increase relative to the blood pool 50 minutes after injection.

We believe our study results represent initial proof-of-concept data for collagen targeting, and further preclinical efficacy and toxicity studies are warranted. Although our study was not designed to address toxicity, no adverse effects such as changes in heart rate were observed during or after injection of EP-3533.

Practical application: A collagentargeting T1-shortening agent, EP-3533, generates positive contrast that may be broadly useful for imaging fibrosis. In addition to being useful for studying the evolution of postinfarction myocardial scarring, EP-3533 may be valuable for detecting and evaluating a broad array of diseases, including hepatic and pulmonary fibroses.

\section{References}

1. Caulfield JB, Borg TK. The collagen network of the heart. Lab Invest 1979;40:364-372.

2. LeGrice IJ, Smaill BH, Chai LZ, et al. Laminar structure of the heart: ventricular myocyte arrangement and connective tissue architecture in the dog. Am J Physiol 1995; 269(2 pt 2):H571-H582.

3. Costa KD, Takayama Y, McCulloch AD, Covell JW. Laminar fiber architecture and three-dimensional systolic mechanics in canine ventricular myocardium. Am J Physiol 1999;276(2 pt 2):H595-H607.
4. Weber KT, Sun Y, Tyagi SC, Cleutjens JP. Collagen network of the myocardium: function, structural remodeling and regulatory mechanisms. J Mol Cell Cardiol 1994;26: $279-292$.

5. Lopez B, Gonzalez A, Querejeta R, Larman M, Diez J. Alterations in the pattern of collagen deposition may contribute to the deterioration of systolic function in hypertensive patients with heart failure. J Am Coll Cardiol 2006;48:89-96.

6. Shirani J, Pick R, Roberts WC, Maron BJ. Morphology and significance of the left ventricular collagen network in young patients with hypertrophic cardiomyopathy and sudden cardiac death. J Am Coll Cardiol 2000; $35: 36-44$.

7. Tanaka M, Fujiwara H, Onodera T, Wu DJ, Hamashima Y, Kawai C. Quantitative analysis of myocardial fibrosis in normals, hypertensive hearts, and hypertrophic cardiomyopathy. Br Heart J 1986;55:575-581.

8. Weisman HF, Healy B. Myocardial infarct expansion, infarct extension, and reinfarction: pathophysiologic concepts. Prog Cardiovasc Dis 1987;30:73-110.

9. Ross AJ, Yang Z, Berr SS, et al. Serial MRI evaluation of cardiac structure and function in mice after reperfused myocardial infarction. Magn Reson Med 2002;47:1158-1168.

10. Epstein FH, Yang Z, Gilson WD, Berr SS, Kramer CM, French BA. MR tagging early after myocardial infarction in mice demonstrates contractile dysfunction in adjacent and remote regions. Magn Reson Med 2002; 48:399-403.

11. Caravan P, Das B, Dumas S, et al. Collagentargeted MRI contrast agent for molecular imaging of fibrosis. Angew Chem Int Ed Engl 2007;46:8171-8173.

12. Rohrer M, Bauer H, Mintorovitch J, Requardt M, Weinmann HJ. Comparison of magnetic properties of MRI contrast media solutions at different magnetic field strengths. Invest Radiol 2005;40:715-724.

13. Berr SS, Roy RJ, French BA, et al. Black blood gradient echo cine magnetic resonance imaging of the mouse heart. Magn Reson Med 2005;53:1074-1079.

14. Yang Z, Berr SS, Gilson WD, Toufektsian MC, French BA. Simultaneous evaluation of infarct size and cardiac function in intact mice by contrast-enhanced cardiac magnetic resonance imaging reveals contractile dysfunction in noninfarcted regions early after myocardial infarction. Circulation 2004;109: 1161-1167.

15. French BA, Beyers RJ, Sureau FC, et al. Imaging infarct expansion in mice using a contrast-enhanced inversion recovery MRI sequence. J Cardiovasc Magn Reson 2005; 7 : $172-173$.

16. Simonetti OP, Kim RJ, Fieno DS, et al. An improved MR imaging technique for the visualization of myocardial infarction. Radiology 2001;218:215-223.

17. Dolber PC, Spach MS. Picrosirius red stain ing of cardiac muscle following phosphomolybdic acid treatment. Stain Technol 1987; 62:23-26.

18. Caravan P. Strategies for increasing the sensitivity of gadolinium based MRI contrast agents. Chem Soc Rev 2006;35:512-523.

19. Chello M, Mastroroberto P, Romano R Perticone F, Marchese AR. Collagen network remodelling and left ventricular function in constrictive pericarditis. Heart 1996;75:184-189.

20. Jugdutt BI, Joljart MJ, Khan MI. Rate of collagen deposition during healing and ventricular remodeling after myocardial infarction in rat and dog models. Circulation 1996; 94:94-101.

21. Kim RJ, Fieno DS, Parrish TB, et al. Relationship of MRI delayed contrast enhancement to irreversible injury, infarct age, and contractile function. Circulation 1999;100: 1992-2002.

22. Choi CJ, Haji-Momenian S, Dimaria JM, et al. Infarct involution and improved function during healing of acute myocardial infarction: the role of microvascular obstruction. J Cardiovasc Magn Reson 2004;6:917-925. 